\title{
CONTENIDO DE GLUCÓSIDOS EN FUNCIÓN DE DEFICIENCIAS NUTRICIONALES EN Stevia rebaudiana BERT.
}

\section{GLYCOSIDE CONTENTS DEPENDING ON THE NUTRIENT DEFICIENCIES IN Stevia rebaudiana BERT.}

\author{
Alfredo Jarma O. ${ }^{1}$ Enrique Combatt C. ${ }^{2}$, Janer Polo S. ${ }^{3}$
}

\begin{abstract}
${ }^{1}$ Ingeniero Agrónomo - Ph.D. Fisiología Vegetal. Profesor Titular Universidad de Córdoba. Carrera 11 No. $62 B$ - 47 - Barrio La Castellana, Montería (Córdoba). E-mail: ajarma@sinu.unicordoba.edu.co. ${ }^{2}$ Ingeniero Agrónomo - Ph.D. Manejo de Suelos y Nutrición de Cultivos. Profesor Titular Universidad de Córdoba. E-mail: ecombatt@sinu.unicordoba. edu.co - 3 Ingeniero Agrónomo - M.Sc. en Biotecnología. Asistente de Investigación. Universidad de Córdoba. E-mail: genomajpolo@gmail.com.
\end{abstract}

Rev. U.D.C.A Act. \& Div. Cient. 15(1): 107 - 116, 2012

\section{RESUMEN}

A nivel mundial, los reportes de los requerimientos nutricionales de estevia (Stevia rebaudiana Bert.), así como de los efectos que las deficiencias de los elementos minerales tienen sobre el contenido de los principales edulcorantes de la hoja, son escasos. El presente trabajo, se realizó en Casa de Vegetación de la Facultad de Ciencias Agrícolas, de la Universidad de Córdoba (Montería, Colombia), con el objetivo de determinar el efecto de las deficiencias nutricionales de N, P, K, Ca, S, Mg, Fe, B, Cu y Zn sobre la concentración de glucósidos totales de la hoja de estevia y, en particular, del rebaudiósido A (RebA), en el genotipo Morita 2. Así mismo, se determinó quincenalmente el contenido de N, P, K, Ca y Mg de raíces, de tallo y de hojas en dos genotipos: Morita 1 (M1) y Morita 2 (M2), hasta el inicio de floración y se estimó la extracción de estos elementos, para una siembra comercial. Los resultados más relevantes indicaron que la concentración de glucósidos totales no se afectó por las deficiencias de nutrientes, aunque la concentración de RebA sólo se redujo ante deficiencias de P, S, K y Cu. La distribución diferencial de los elementos mayores en los órganos de la planta permitió establecer la extracción de estos en $\mathrm{kg} / \mathrm{ha}$, que fue respectivamente para $M 1$ y $M 2$ de 24,9 y 30,8 (N); 4,8 y 6,3 (P); 23,7 y 29,9 (K); 9,7 y 10,8 (Ca) y 6,7 y 6,3 (Mg).

Palabras clave: Estevia, estrés nutricional, esteviósidos, rebaudiósido A, nutrición mineral.

\section{SUMMARY}

Worldwide, there are few reports of stevia's (Stevia rebaudiana Bert.) nutritional requirements, as well as the effects of deficiencies of mineral elements on the content of the main sweetener of the leaf. The study was carried in the greenhouse of Agricultural Sciences Faculty, University of Córdoba (Monteria - Colombia), in 2008. The objective was to determine the effect of nutritional deficiencies of $N$, $\mathrm{P}, \mathrm{K}, \mathrm{Ca}, \mathrm{S}, \mathrm{Mg}, \mathrm{Fe}, \mathrm{B}, \mathrm{Cu}$, and $\mathrm{Zn}$ on the concentration of total glycosides of the stevia leaf, in particular of rebaudioside A (RebA) in the Morita 2 genotype. Also, every 15 days, the content of $\mathrm{N}, \mathrm{P}, \mathrm{K}, \mathrm{Ca}$ and $\mathrm{Mg}$ from the roots, stems and leaves in two genotypes, Morita 1 (M1) and Morita 2 (M2), until the time of flowering onset was estimated. The removal of these elements for commercial planting was also evaluated. The main results indicated that the concentration of total glycosides was not affected by nutrient deficiencies, although the concentration of RebA was reduced, only due to deficiencies in $\mathrm{P}, \mathrm{S}, \mathrm{K}$ and $\mathrm{Cu}$. The differential distribution among genotypes of the major elements in the plant organs allowed to establish their extraction, expressed as $\mathrm{kg} / \mathrm{ha}$, respectively for M1 and M2 of 24.9 and $30.8(\mathrm{~N}), 4.8$ and $6.3(\mathrm{P}), 23.7$ and $29.9(\mathrm{~K}), 9.7$ and $10.8(\mathrm{Ca})$ and 6.7 and $6.3(\mathrm{Mg})$.

Key words: Stevia, nutritional stress, steviosides, rebaudioside A, mineral nutrition. 


\section{INTRODUCCIÓN}

El género Stevia es exclusivo del continente americano y se distribuye desde el sudeste de Estados Unidos hasta Argentina. Estevia (Stevia rebaudiana Bert.) es nativa del Norte de Paraguay, empleada por mucho tiempo por los indígenas nativos en tés y preparaciones de alimentos (Dacome et al. 2005), aunque sus principales propiedades son como edulcorante y medicinal.

La información científica indica que la cantidad de los dos principales glucósidos, esteviósido y rebaudiósido A (Brandle \& Telmer, 2007), dependen, en gran medida, del genotipo y las condiciones ambientales (Gardana et al. 2010), del manejo de prácticas agronómicas, como la nutrición mineral (Nepovim et al. 1998; Geuns, 2003; Das et al. 2007) y el riego (Fronza \& Folegatti, 2003); estas últimas prácticas, además, pueden llegar a aumentar los rendimientos de hoja seca por hectárea, a 2000-3000kg y el contenido de glucósidos de 15-20\% (Barathi, 2002).

Los estudios que abordan el tema de la nutrición mineral en estevia en el mundo son escasos (Ramesh et al. 2006). Se pueden resaltar trabajos desarrollados en India, en Brasil y en Colombia; por ejemplo, en India, se demostró el efecto positivo de la combinación de bacterias solubilizadoras de fósforo, micorrizas y Azotobacter sobre el incremento de biomasa de plantas completas (Das et al. 2008). Trabajos de fertilización de síntesis química en Brasil, reportaron que, para obtener una tonelada de hoja seca por hectárea, se requieren $130 \mathrm{~kg} / \mathrm{ha}$, de $\mathrm{N} ; 18,8$, de P; 131,5, de K; 43,7, de Ca; 8,3, de Mg y 9,7, de S (Utumi et al. 1999; De Lima et al. 1997a; De Lima \& Malavolta, 1997). En Colombia, investigaciones adelantadas por Jarma, en 2008, reportan requerimientos de $70,9.2$ y $32 \mathrm{~kg} / \mathrm{ha}$ de $\mathrm{N}, \mathrm{P}$ y $\mathrm{K}$, respectivamente, para obtener una tonelada de hoja seca por hectárea, bajo condiciones controladas de casa de vegetación y de suelos de textura arcillosa.

El efecto de las deficiencias nutricionales sobre la cantidad de glucósidos, también ha sido pobremente documentado. De Lima et al. (1997b) reportan que solamente una deficiencia severa de calcio ocasionó reducciones significativas en la concentración foliar de los edulcorantes.

Considerando el vacío que existe sobre requerimientos y distribución de nutrientes en estevia, el presente trabajo fue desarrollado con dos propósitos: el primero, determinar el efecto de las deficiencias nutricionales de $\mathrm{N}, \mathrm{P}, \mathrm{K}, \mathrm{Ca}, \mathrm{S}, \mathrm{Mg}$, $\mathrm{Fe}, \mathrm{B}, \mathrm{Cu}$ y $\mathrm{Zn}$, sobre la concentración total de glucósidos, el contenido relativo de rebaudiósido A (RebA) y la relación entre éste y otros glucósidos (OG) en hojas de Morita 2 y, el segundo, evaluar el contenido de elementos mayores en raíces, en tallos y en hojas, hasta la primera cosecha de hojas de los genotipos Morita 1 (M1) y Morita 2 (M2), únicos materiales conocidos comercialmente en Colombia.

\section{MATERIALES Y MÉTODOS}

Localización y material experimental: Se desarrollaron dos trabajos durante el segundo semestre de 2008. El ensayo de deficiencias nutricionales, se realizó bajo condiciones controladas, en invernaderos experimentales $\left(58 \%\right.$ de $\mathrm{HR}, 30{ }^{\circ} \mathrm{C}$ de temperatura media y $1000 \mu$ moles de fotones fotosintéti$\cos \mathrm{m}^{-2} \mathrm{~s}^{-1}$ ) y el de distribución de nutrientes en campo, en la Facultad de Ciencias Agrícolas, de la Universidad de Córdoba (Montería-Colombia), con coordenadas geográficas de $8^{\circ}$ 52 ' LN y $76^{\circ} 48^{\prime}$ LO, 13 msnm y temperatura media anual de $28^{\circ} \mathrm{C}$. Las características del sustrato utilizado en el primer ensayo fueron arena pasada por tamiz, lavada y tratada con $\mathrm{HCl}$ concentrado comercial, para la purificación del material. Esta arena permaneció en el ácido por 24 horas; después fue sometida a lavados sucesivos con agua corriente, para retirar el exceso de ácido, hasta que el $\mathrm{pH}$ alcanzó valores de pH cercanos a 6,0. La segunda investigación fue realizada en suelo con composición de arcillas $2: 1$, que en su totalidad están compuesta por arcillas ilita y montmorillonita.

Contenido de glucósidos en función de deficiencias nutricionales: Se establecieron once tratamientos bajo un diseño completamente al azar con tres repeticiones. Cada unidad experimental (UE) correspondió a 15 plantas de M2, sembradas con la misma edad fisiológica (esquejes con 6 pares de hojas), a las cuales, se aplicó,, tres veces por semana, una solución nutritiva con el elemento faltante y un testigo experimental (solución completa sin deficiencias de elementos). La denominación de los tratamientos fue la siguiente: T1 (testigo experimental TE), T2 (-N), T3 (-K), T4 (-Ca), T5 (-Mg), T6 (-P), T7 (-S), T8 (-Fe), T9 (-B), T10 (-Cu), T11 (-Zn). Aunque se incluyó el tratamiento sin $M n$, no fue posible obtener lectura de glucósidos en las muestras de éste, por escasez de material vegetal. Como solución nutritiva, se usó la solución madre de Johnson, adaptada por De Lima \& Malavolta, en 1997 (Tabla 1), a la cual, se le retiraron los elementos respectivos, de acuerdo a cada tratamiento. Antes de establecer el experimento, los esquejes se colocaron en agua deionizada, durante siete días. La siembra, se realizó en macetas plásticas de 5L de capacidad, que contenían un sustrato de arena inerte lavada sin materia orgánica, con las siguientes características: $\mathrm{pH}$, de 6,92; $22 \mathrm{mg} \mathrm{kg}^{-1}$, de S; 5,6, de P; 0,9, de Cu; 76, de Fe; 0,4, de Zn y 79,8, de Mn; 2,1 cmol $_{\mathrm{c}} \mathrm{kg}^{-1}$, de Ca; 0,7, de Mg; 0,06, de K; 0,11, de Na y 3,0, de CIC. El muestreo foliar, se realizó a diez plantas escogidas al azar de cada UE, a los 75 días después del trasplante (ddt), justo al momento de iniciar floración. 
Tabla 1. Solución madre de Johnson (adaptada por De Lima \& Malavolta, en 1997).

\begin{tabular}{|c|c|c|}
\hline Solución & Compuesto & Concentración \\
\hline $\mathrm{A}$ & $\mathrm{Ca}(\mathrm{NO})_{3} 4 \mathrm{H}_{2} \mathrm{O}$ & $1 M\left(236 \mathrm{~g} \mathrm{~L}^{-1}\right)$ \\
\hline $\mathrm{B}$ & $\mathrm{KNO}_{3}$ & $1 \mathrm{M}\left(101 \mathrm{~g} \mathrm{~L}^{-1}\right)$ \\
\hline $\mathrm{C}$ & $\mathrm{MgSO}_{4} 7 \mathrm{H}_{2} \mathrm{O}$ & $1 M\left(246.5 \mathrm{~g} \mathrm{~L}^{-1}\right)$ \\
\hline $\mathrm{D}$ & $\mathrm{K}_{2} \mathrm{HPO}_{4}$ & $1 M\left(136 \mathrm{~g} \mathrm{~L}^{-1}\right)$ \\
\hline $\mathrm{E}$ & $\mathrm{FeSO}_{4}$ & $25 \mathrm{~g} \mathrm{~L}^{-1}$ \\
\hline $\mathrm{E}$ & $\mathrm{NaOH}$ & $14 \mathrm{~g} \mathrm{~L}^{-1}$ \\
\hline $\mathrm{E}$ & Etilenodiaminetracetato de Sodio & $26 \mathrm{~g} \mathrm{~L}^{-1}$ \\
\hline $\mathrm{F}$ & $\mathrm{MnCl}_{4} \mathrm{H}_{2} \mathrm{O}$ & $1,82 \mathrm{~g} \mathrm{~L}^{-1}$ \\
\hline G & $\mathrm{H}_{3} \mathrm{BO}_{3}$ & $2,86 \mathrm{~g} \mathrm{~L}^{-1}$ \\
\hline $\mathrm{H}$ & $\mathrm{ZnSO}_{4} 7 \mathrm{H}_{2} \mathrm{O}$ & $0,22 \mathrm{~g} \mathrm{~L}^{-1}$ \\
\hline I & $\mathrm{CuSO}_{4} 5 \mathrm{H}_{2} \mathrm{O}$ & $0,88 \mathrm{~g} \mathrm{~L}^{-1}$ \\
\hline $\mathrm{J}$ & $\mathrm{NaNO}_{3}$ & $1 M\left(85,01 \mathrm{~g} \mathrm{~L}^{-1}\right)$ \\
\hline $\mathrm{K}$ & $\mathrm{MgCl}_{2}$ & $1 \mathrm{M}\left(9523 \mathrm{~g} \mathrm{~L}^{-1}\right)$ \\
\hline $\mathrm{L}$ & $\mathrm{Na}_{2} \mathrm{SO}_{4}$ & $1 M\left(142,06 \mathrm{~g} \mathrm{~L}^{-1}\right)$ \\
\hline M & $\mathrm{NaH}_{2} \mathrm{PO}_{4} \mathrm{H}_{2} \mathrm{O}$ & $1 \mathrm{M}\left(138,01 \mathrm{~g} \mathrm{~L}^{-1}\right)$ \\
\hline $\mathrm{N}$ & $\mathrm{CaCl}_{2}$ & $1 \mathrm{M}\left(110,99 \mathrm{~g} \mathrm{~L}^{-1}\right)$ \\
\hline $\mathrm{O}$ & $\mathrm{KCl}$ & $1 \mathrm{M}\left(74,55 \mathrm{~g} \mathrm{~L}^{-1}\right)$ \\
\hline
\end{tabular}

La concentración de glucósidos totales, se estimó en porcentaje de peso seco del promedio de todas las hojas de la planta, por el método de cromatografía líquida de alta eficacia; se calculó utilizando, en la fase móvil, una mezcla de acetonitrilo y de agua (80:20), con una columna Kromasil $100-5 \mathrm{HN}_{2}$ (método isocrático) y un patrón de $90 \%$ de pureza, considerada como control. Estos análisis fueron realizados por el Laboratorio de Control de Calidad, de la empresa Prodalysa Ltda (Chile). Solo se consideró la presencia de RebA en función de glucósidos totales (GT), por ser de mayor importancia económica (Liu et al. 2010). La diferencia entre GT y RebA, se asumió como contenido de OG, para efectos de establecer la relación RebA/OG. Los datos obtenidos fueron analizados bajo el software estadístico Statistical Análisis System (SAS) versión 9.1 y las pruebas de comparación de media, se estimaron al 5\%, bajo la prueba de DMS.

Distribución de nutrientes en función del órgano y la edad: En este segundo experimento, a partir de los $15 \mathrm{ddt}$, se ejecutaron de cuatro a cinco muestreos quincenales, en los cuales, fueron estimados los contenidos nutricionales en hojas, en tallos y en raíces de plantas completas de $M 1$ y M2. El último muestreo, se llevó a cabo al inicio de floración, que se presentó a los 60 ddt, para M1 y 75, para M2. En este experimento, se establecieron en campo 1260 plantas de cada genotipo sembrados, a $0,4 \mathrm{~m}$, entre surcos y $0,2 \mathrm{~m}$, entre plantas.

Los muestreos destructivos, se practicaron en diez plantas de cada genotipo, escogidas al azar y en competencia intra-específica. Para cuantificar $\mathrm{Ca}, \mathrm{K}, \mathrm{Mg}$ y P, inicialmente, se calcinaron las muestras de tejido foliar, para obtener los óxidos o las sales de los elementos, que fueron posteriormente disueltos con ácido clorhídrico 1:1 y llevados a un volumen conocido. La determinación, se efectuó por espectrofotometría de absorción atómica (EAA) que, para el caso del $\mathrm{Ca}, \mathrm{K}$ y Mg, se realizó una dilución previa en la que se adiciona lantano y sodio, como supresores de ionización. Los análisis, se hicieron en el Laboratorio de Suelos, de la Universidad Nacional de Colombia, Sede Bogotá.

\section{RESULTADOS Y DISCUSIÓN}

Contenido de glucósidos en función de deficiencias nutricionales: Estavariablenosevioafectadasignificativamente $(p>0.05)$ por ninguno de los tratamientos en los que se indujo deficiencia nutricional (Tabla 2). Los resultados indicaron que la concentración total de glucósidos presentes en las hojas, 
Tabla 2. Significancia estadística de variables de calidad en hoja de estevia en función de once tratamientos de deficiencias nutricionales.

\begin{tabular}{|c|c|c|c|c|}
\hline \multirow{2}{*}{ Fuente de Variación } & \multirow{2}{*}{ Gl } & \multicolumn{3}{|c|}{ Cuadrado Medio del Error } \\
\hline & & GT & RebA & Relación RebA/OG \\
\hline Tratamientos & 10 & $4,12 \mathrm{NS}$ & $8,97 * *$ & $0,2593 * *$ \\
\hline Media & & $\begin{array}{c}8,57 \\
\text { (dag kg-1 en hoja seca) }\end{array}$ & $\begin{array}{c}76,2 \\
\text { (\% de GT) }\end{array}$ & 3,39 \\
\hline $\mathrm{CV}(\%)$ & & 23,6 & 3,9 & 15,0 \\
\hline
\end{tabular}

$\mathrm{GT}=$ Glucósidos totales; RebA=Rebaudiósido A; $\mathrm{OG}=$ Otros glucósidos; $\mathrm{CV}=$ Coeficiente de variación y gl $=$ Grados de Libertad. NS, $* \mathrm{y} * *=$ no significativo, significativo $(\mathrm{p} \leq 0.05)$ y altamente significativo $(\mathrm{p} \leq 0.01)$.

con un promedio de $8,57 \%$ fue bajo al ser comparados con resultados reportados por Hearn \& Subedi (2006). Aunque existen pocas referencias mundiales, resultados similares fueron reportados por De Lima \& Malavolta (1997), quienes al evaluar el efecto de las deficiencias de N, P, K, Ca, Mg, S, B y Zn sobre la concentración de glucósidos, sólo encontraron efectos importantes en la reducción del edulcorante ante deficiencias severas de $\mathrm{Ca}$; sin embargo, los autores no discuten sus resultados.

Por su parte, Utumi et al. (1999) hallaron que sólo las deficiencias de $\mathrm{K}$, Ca y $\mathrm{S}$ disminuyeron la concentración de glucósidos (dag kg-1 de hoja seca) y estas deficiencias, a excepción de la de P, disminuyeron el contenido total de glucósidos en la planta; los autores concluyeron que esto último fue una consecuencia directa de la reducción en la biomasa de la misma.

Aunque la nutrición juega un papel definitivo en la síntesis de estas moléculas edulcorantes (Epstein \& Bloom, 2005; Jarma, 2008; Das etal. 2008) es posible que otras variables, no consideradas en el estudio, como la luz (Ramesh et al. 2006; Allam et al. 2001), la temperatura (Mizukami et al. 1983), el agua (Fronza \& Folegatti, 2003), las técnicas de manejo (Geuns, 2003) y la edad de las plantas (De Lima \& Malavolta, 1997), entre otros factores, fueron más determinantes en el contenido de glucósidos que las respuestas observadas en este trabajo, por efecto de las deficiencias.

Un resultado interesante del presente trabajo permite establecer que, a pesar de no encontrarse variaciones en la concentración de GT, la concentración del RebA sí varió significativamente $(p \leq 0.01)$, cuando se indujeron las deficiencias (Figura 1). Estos resultados, se pueden considerar novedosos, porque la mayoría de estudios publicados de nutrición y de cantidad de glucósidos, siempre se refieren a glucósidos totales sin discriminarlos (De Lima et al. 1997b; Nepovim et al. 1998; Utumi et al. 1999).

La figura 1 muestra que la concentración de RebA, se redujo significativamente $(p \leq 0.05)$ respecto al $T E$, cuando se indujeron deficiencias de $\mathrm{P}, \mathrm{S}, \mathrm{KyCu}$, pero que las deficiencias de N, Ca, Mg, Fe, B y Zn, al parecer, no causan decrecimos importantes en este glucósido. Probablemente, estos resultados se explican por el estado juvenil de las plantas que no alcanzan a mostrar el efecto de las deficiencias, aunque resultados similares reportaron Bondarev et al. (2003), al no encontrar efecto de elementos nutricionales sobre el balance de glucósidos en las hojas de estevia a nivel in vitro. La concentración de este glucósido que se presentó en el tratamiento con deficiencia de $\mathrm{S}$ son coincidentes con los de Utumi et al. (1999); sin embargo, es importante considerar que los autores citados, evaluaron la concentración total de glucósidos, donde además de RebA, estarían presentes otras moléculas edulcorantes en menor proporción y lo que haría diferencia con los resultados observados en este estudio.

Es importante recalcar que uno de los criterios mundiales para determinar la calidad de la hoja está dado por la cantidad de RebA presente en esta parte de la planta, ya que los extractos considerados como más valiosos, son aquellos que tienen RebA como el principal componente (Dacome et al. 2005). Esto es debido a sus características organolépticas y fisicoquímicas, al manifestar el mejor perfil 


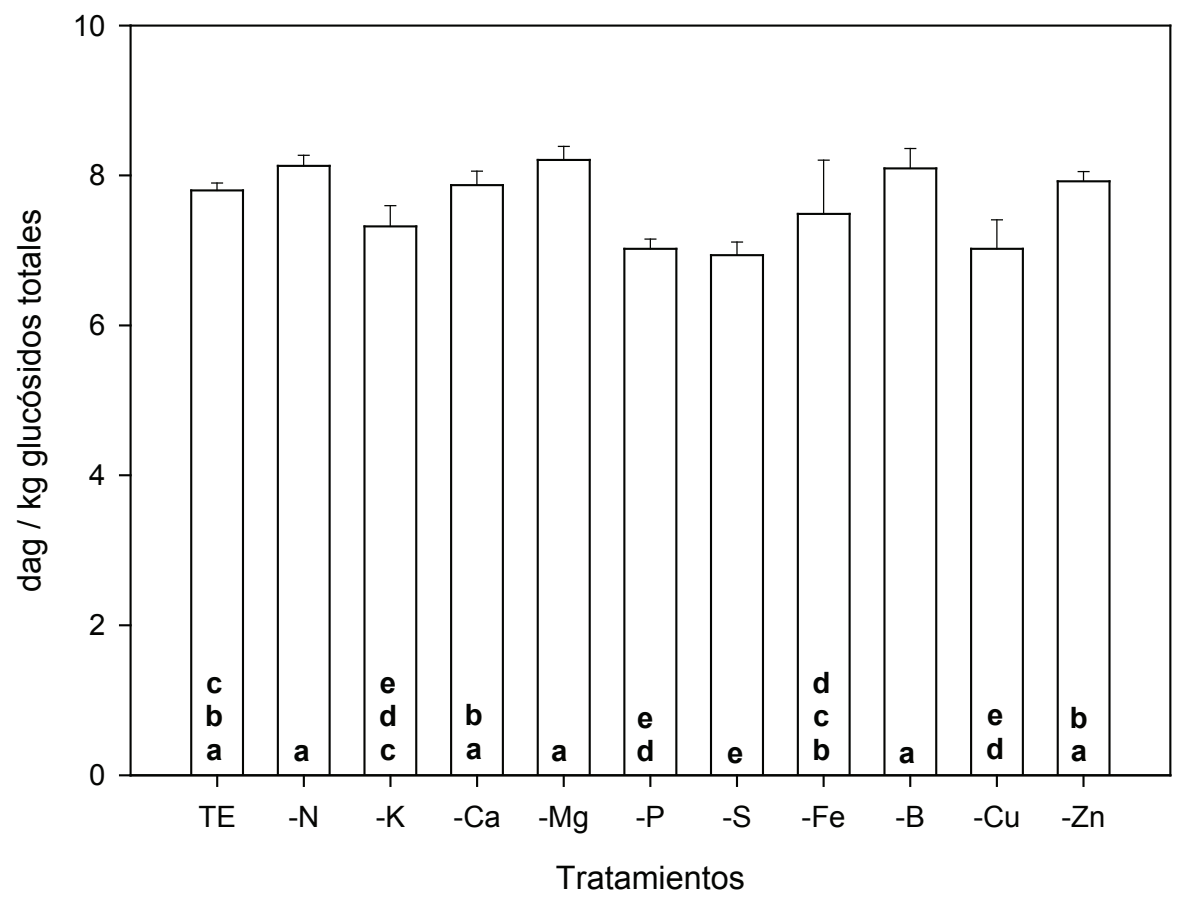

Figura 1. Concentración de rebaudiósido A en hojas de estevia sometidas a deficiencias nutricionales. Barras con la misma letra no difieren estadísticamente, de acuerdo al test protegido de DMS (5\%); TE = Desviación estándar.

de sabor respecto a los otros glucósidos y tener una mayor solubilidad en agua, lo que permite una mayor variedad de formulaciones (Brandle, 1999; Brandle et al. 2002).

Al considerar la relación RebA/OG, los resultados indicaron que se presentaron diferencias estadísticas $(\mathrm{p} \leq 0.01)$, en función de las deficiencias nutricionales (Tabla 3).

Con base en los reportes mundiales, la relación RebA/OG observada en el presente estudio $(3,39 \pm 0,3)$, se estima como alta, si se considera que dentro de OG se incluye, principalmente, el esteviósido (St), ya que, por lo general, se informan relaciones RebA/St de 0,2 a 0,4 (Erkucuk et al. 2009) 0,5 (Dacome et al. 2005); 0,5 a 0,6 (Midmore \& Rank, 2002). Estos últimos autores indican que la relación RebA/St es muy importante, por que el St imparte una característica un poco amarga al sabor de los extractos crudos, la cual, no es percibida cuando RebA está en igual o mayor cantidad que St.

Los resultados de la tabla 3 muestran que las deficiencias de $\mathrm{K}, \mathrm{Cu}, \mathrm{P}$ y $\mathrm{S}$ redujeron significativamente $(\mathrm{p} \leq 0.05)$ la relación RebA/OG, sugiriendo que estas carencias reducirían, principalmente, el contenido de RebA o, también, que aumentarían el contenido de OG, aunque con base en las respuestas discutidas en acápites anteriores es probable que la reducción del RebA, ante deficiencias de estos elementos,

Tabla 3. Relación RebA/OG en hojas de estevia sometidas a deficiencias nutricionales.

\begin{tabular}{|c|l|}
\hline Tratamiento & Promedio \pm SD \\
\hline- Mg & $4,61 \pm 0,53 \mathrm{a}$ \\
\hline- N & $4,35 \pm 0,42 \mathrm{ab}$ \\
\hline- B & $4,31 \pm 0,81 \mathrm{ab}$ \\
\hline- Zn & $3,82 \pm 0,31 \mathrm{abc}$ \\
\hline- Ca & $3,72 \pm 0,42 \mathrm{bc}$ \\
\hline TE & $3,55 \pm 0,20 \mathrm{bcd}$ \\
\hline- Fe & $3,17 \pm 1,02 \mathrm{cde}$ \\
\hline- K & $2,75 \pm 0,37 \mathrm{def}$ \\
\hline$-\mathrm{Cu}$ & $2,39 \pm 0,43 \mathrm{ef}$ \\
\hline$-\mathrm{P}$ & $2,36 \pm 0,15 \mathrm{ef}$ \\
\hline$-\mathrm{S}$ & $2,27 \pm 0,19 \mathrm{f}$ \\
\hline
\end{tabular}

$\mathrm{TE}=$ Testigo experimental (solución sin deficiencias nutricionales). $\mathrm{SD}=$ desviación estándar. Promedios con la misma letra son iguales estadísticamente, según la prueba de DMS (5\%). 
sea lo más probable. Adicionalmente, no ha sido demostrado que la deficiencia de un elemento derive en el aumento de cualquiera de los glucósidos presentes en la hoja, máxime si se considera su similitud estructural, pues su diferencia radica solamente en la presencia de una glucosa (Carakostas et al. 2008). De igual forma, al parecer ninguna de las deficiencias estudiadas registró cambios positivos en la relación RebA/ OG respecto al TE, a excepción de la deficiencia de Mg.

Con relación a la variable RebA/OG son pocas las referencias que existen para comparar los resultados de esta investigación, pero es probable que las deficiencias nutricionales generen un efecto diferencial indirecto sobre la síntesis de RebA u OG, ya que la presencia o ausencia de los elementos está ligada a varios cofactores enzimáticos de varias glucosiltransferasas, cuya actividad deriva en elaboración de moléculas de diferentes glucósidos de esteviol por glucosilaciones diferenciales (Richman et al. 2005); estas glucosilaciones proceden en propiedades organolépticas distintivas; por ejemplo, se reporta que St es 143 veces más dulce que la sacarosa con base en el peso y que el RebA es 242 veces (Brandle \& Telmer, 2007).

Es importante indicar que no sorprende el consistente efecto mostrado por el P, en los resultados de este estudio, dado su papel definitivo como constituyente de la mayoría de moléculas que intervienen en la síntesis del kaureno, estructura molecular precursora de glucósidos por la ruta del metil-eritritol fosfato, a partir del ácido pirúvico y del gliceraldehído 3 fosfato (Azcón-Bieto \& Talón, 2008; ÁlvarezGarcía et al. 2005).

Distribución de nutrientes en función del órgano y la edad: En estos resultados, se muestra la tendencia de la distribución de los elementos mayores en función del genotipo y la edad fisiológica de hojas, tallos y raíces (Figura 2).

Nitrógeno: La dinámica de este elemento fue similar para M1 y M2, aunque las eficiencias en el uso de este elemento difieren de manera importante entre especies y dentro de éstas (Fan et al. 2005). En los tres órganos, se observó una tendencia decreciente en el tiempo, a excepción de las hojas donde, en promedio para los dos genotipos, a los 30ddt, se presenta un leve incremento de alrededor del $111 \%$ que, posteriormente, decrece hasta el inicio de la floración, llegando a un 65\% del N inicial (Figura 2).

Probablemente, esta disminución sea debida a una redistribución del nitrógeno hacia las estructuras reproductivas, de próxima aparición, pero que no fueron consideradas en el trabajo, donde sólo se evaluó la distribución de nutrientes hasta el momento de la cosecha comercial (inicio de primordios florales). Tendencias similares reportaron De Lima et al. (1997a), en un trabajo con estevia en Brasil, aunque estos autores sí condujeron la investigación hasta la presencia de flores e, incluso, semillas, órganos que determinaron de manera importante una migración del $\mathrm{N}$ desde las hojas.

Fósforo: La figura 2 muestra que, en términos generales, la concentración tisular de $\mathrm{P}$ se mantuvo relativamente constante a través del tiempo, siendo la hoja el órgano de mayor concentración del elemento. Al final del ciclo, los niveles foliares de $P$ fueron de 3,9 y $3,1 \mathrm{~g} \mathrm{~kg}^{-1}$ de masa seca para M1 y M2, respectivamente. M1 registró valores más altos de $\mathrm{P}$ en hoja y en tallo, en tanto que en la raíz fueron más bajos respecto a M2. De Lima et al. (1977a) reportaron concentraciones de $\mathrm{P}$ en el tallo de $2,5 \mathrm{~g} \mathrm{~kg}^{-1}$, a los $45 \mathrm{ddt}$, valores que son similares a los hallados en este estudio en la misma época (2,7 y 2,3 $\mathrm{g} \mathrm{kg}^{-1}$ para M1 y M2, respectivamente).

De manera similar, el estudio de Brasil encontró concentraciones de $\mathrm{P}$ de $1,6 \mathrm{~g} \mathrm{~kg}^{-1}$ en raíz al final del experimento $(90$ ddt), coincidiendo con los de $M 1$ (1,3g kg-1) y M2 (1,9g kg-1), reportados en este estudio. Siendo las hojas un órgano de mayor tasa de división y de expansión celular que el tallo, así como de mayores tasas de fotosíntesis y de respiración es probable que, dados los requerimientos de energía en éstas, la concentración del $\mathrm{P}$ en estas sea mayor respecto al tallo (Su et al. 2006).

Potasio: En ambos genotipos y en todos los órganos del vegetal, la concentración de $\mathrm{K}$ decreció con el tiempo, registrándose solamente un leve incremento hacia los 60ddt, que luego bajó en el genotipo M2, a los 75ddt (Figura 2). Respecto a las concentraciones iniciales por órgano (hoja 17,5 ; tallo 25,5 y raíz $15,0 \mathrm{~g} \mathrm{~kg}^{-1}$ ) y de manera independiente al genotipo, los niveles de $\mathrm{K}$ cayeron en $24 \%$ (hoja), $48 \%$ (tallo) y $33 \%$ (raíz), siendo el tallo el órgano con mayores eflujos de este elemento. La disminución en la concentración de K en la planta, a través del tiempo, también fue reportada por De Lima et al. (1977a).

Es probable que esta redistribución del K, paralelamente a la del $\mathrm{N}$, se de hacia flores y semillas que estarían a punto de aparecer. Consecuentemente, la hoja sería el órgano que menores niveles de migración presentaría, por la alta actividad fotosintética en pre-floración, donde es requerida una alta eficiencia de $\mathrm{K}$ en la apertura y cierre estomático, para mantener el estado hídrico de la planta y la activación de muchas enzimas esenciales para la fotosíntesis y la respiración (Taiz \& Zeiger, 2006).

Calcio: La concentración de Ca en tejido vegetal registró una tendencia decreciente en los dos genotipos, con respecto a los tiempos de muestreo, como se puede observar en la 

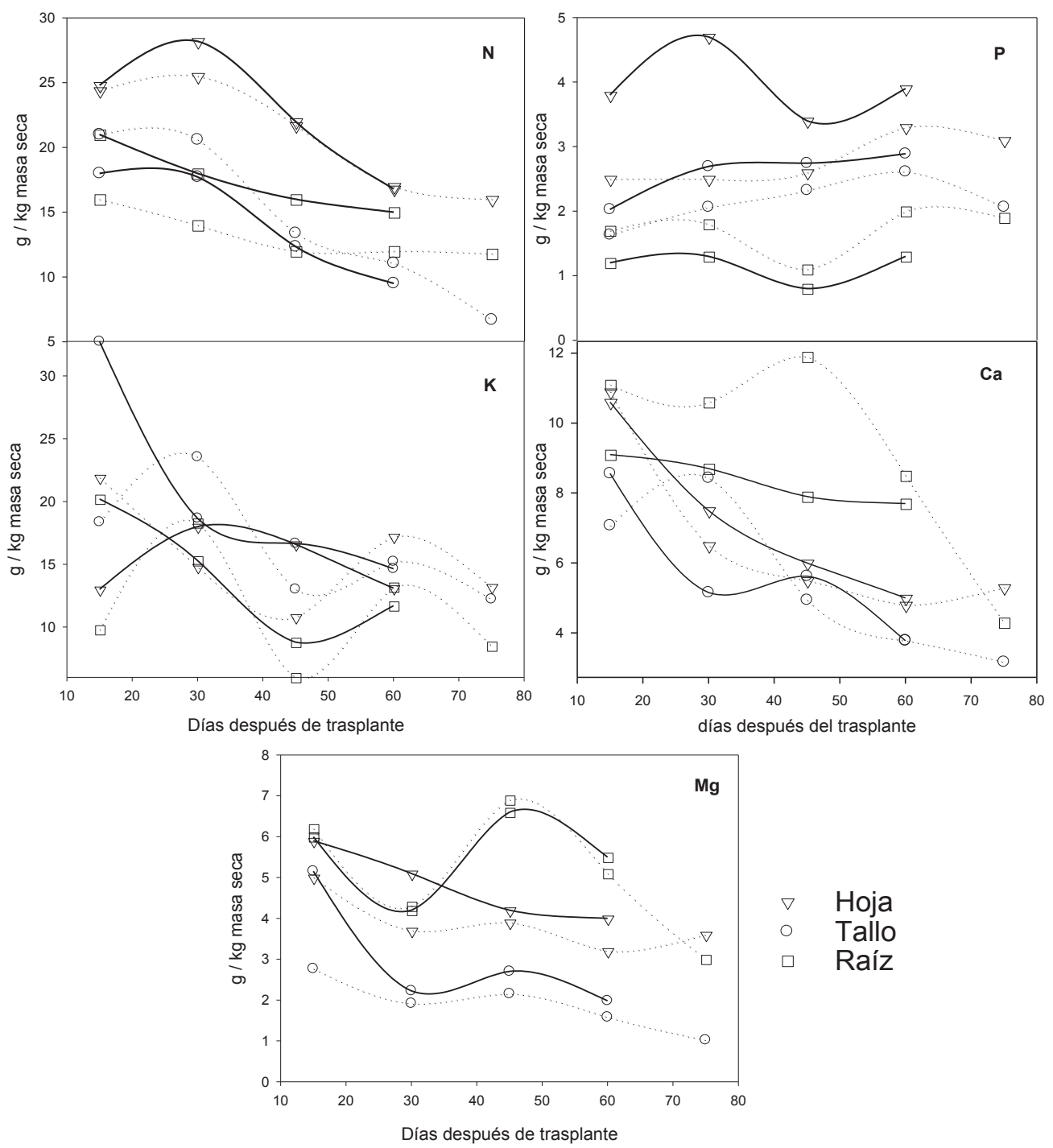

Hoja

Tallo

Raíz

Morita 1

Morita 2

Figura 2. Concentración de elementos mayores ( $\mathrm{g} / \mathrm{kg}$ de masa seca) en tres órganos de dos genotipos de estevia.

figura 2. Los niveles iniciales fueron de alrededor de $9 \mathrm{~g} \mathrm{~kg}^{-1}$ en promedio para todos los órganos y de $4 \mathrm{~g} \mathrm{~kg}^{-1}$ al final del ciclo; sin embargo, al momento de cosecha, la concentración en raíz fue mayor que en el resto de la planta, llegando a representar el $50 \%$ del Ca, presente a los $60 \mathrm{ddt}$, seguido de las hojas (30\%) y, por último, en el tallo (20\%).

En la raíz, los altos niveles de concentración de Ca podrían ser explicados por la eficiente captación de este elemento por las raíces desde la fracción disponible del suelo, pero poca redistribución de este elemento hacia hoja y tallo. De Lima et al. (1997) encontraron una alta presencia de Ca en raíces al final del ciclo y una migración importante desde el tallo; los autores argumentaron que esto era debido, probablemente, a una redistribución hacia órganos reproductivos. De todas maneras, conociendo la poca movilidad de estos elementos y la dificultad que tiene para ser cargado en el floema, White \& Broadley (2003) explican que la migración desde el tallo ocurriría a partir del Ca intercambiable en el xilema y en el apoplasto de las células del tallo, dirigiéndose, principalmente, a las hojas (Marschner, 2002). 
Magnesio: Al observar la concentración del Mg en los tres órganos (Figura 2), se nota un comportamiento similar entre los genotipos y los órganos de la planta, registrándose una leve tendencia decreciente en la raíz, en el tallo y en la hoja: en la raíz, a los $60 \mathrm{ddt}$, fue el órgano donde mayor concentración de este elemento se encontró, con 5,3 $\mathrm{g} \mathrm{kg}^{-1}$; en hoja, $3,6 \mathrm{~g} \mathrm{~kg}^{-1} \mathrm{y}$, por último, en el tallo, $1,8 \mathrm{~g} \mathrm{~kg}^{-1}$.

Las concentraciones de $\mathrm{Mg}$ en los órganos de estevia apreciados en el presente trabajo son más altos que los reportados por De Lima et al. (1997a), quienes informaron concentraciones de 0,$7 ; 1,4$ y $0,6 \mathrm{~g} \mathrm{~kg}^{-1}$, en raíz, en hoja y en tallo, respectivamente, a los 60ddt. La concentración de $\mathrm{Mg}$ en las hojas, que tiende levemente a crecer al final del ciclo en los dos genotipos, podría indicar que este elemento se concentra en este órgano para desempeñar un papel importante en la síntesis de glucósidos de esteviol. En efecto, el $\mathrm{Mg}$ se combina con el ATP, permitiéndole participar en muchas reacciones, como la fosforilación del ácido mevalónico AMV, para formar isopentenil-pirofosfato IPP, primer compuesto isoprenoide en la síntesis de estos glucósidos; seguidamente, en la misma ruta de síntesis, actúa como cofactor de la enzima citoplasmática farnesil pirofosfato sintasa, que cataliza la formación del farnesil pirofosfato (Srivastava, 2002).

Exportación de nutrientes: La tabla 4 registra la masa seca por planta de las hojas a los 60ddt, para M1 y a los 75ddt, para M2, y las concentraciones totales por planta de cada elemento.

Con base en la concentración de los elementos nutricionales en la planta, la producción de hoja seca/planta de M1 y M2 desde el trasplante hasta la primera cosecha de hojas y considerando una población de 100 mil plantas/ha, se estimaría una extracción en $\mathrm{kg} / \mathrm{ha}$ de nutrientes en la época de cosecha de cada genotipo así: 24,9 (N); 4,8 (P); 23,7 (K); 9,7 (Ca) y 6,7 (Mg) para M1y 30,8 (N); 6,3 (P); 29,9 (K); 10,8 (Ca) y 6,3 (Mg) para M2. Obviamente es necesario considerar que en las cosechas sucesivas de la planta estos valores serán más altos.

De los resultados obtenidos, se puede concluir que las deficiencias nutricionales no influyen sobre la concentración

Tabla 4. Masa seca hojas ( $\mathrm{g} /$ planta) y concentración de elementos mayores ( $\mathrm{g} / \mathrm{kg}$ de masa seca) de dos genotipos de estevia.

\begin{tabular}{|c|c|c|c|c|c|c|}
\hline \multirow[t]{2}{*}{ Nutriente } & \multirow{2}{*}{ Genotipo } & \multicolumn{5}{|c|}{ Días después del trasplante } \\
\hline & & 15 & 30 & 45 & 60 & 75 \\
\hline \multirow{3}{*}{ Masa seca hojas/planta (g) } & M1 & 0,88 & 1,67 & 3,22 & 6,08 & \\
\hline & M2 & 0,91 & 1,93 & 3,68 & 7,65 & 9,06 \\
\hline & & \multicolumn{5}{|c|}{$\begin{array}{c}\text { Concentración de elementos } \\
(\mathrm{g} / \mathrm{kg} \text { masa seca })\end{array}$} \\
\hline \multirow{2}{*}{$\mathrm{N}$} & M1 & 63,8 & 63,9 & 50,3 & 41,3 & \\
\hline & M2 & 61,4 & 60,1 & 47,1 & 40,1 & 34,5 \\
\hline \multirow{2}{*}{$\mathrm{P}$} & M1 & 7,0 & 8,7 & 6,9 & 8,1 & \\
\hline & M2 & 5,8 & 6,4 & 6,0 & 7,9 & 7,1 \\
\hline \multirow{2}{*}{$\mathrm{Ca}$} & M1 & 32,3 & 21,4 & 19,5 & 16,5 & \\
\hline & M2 & 29,1 & 25,5 & 22,3 & 17,1 & 12,8 \\
\hline \multirow{2}{*}{$\mathrm{K}$} & M1 & 66,0 & 52,0 & 42,1 & 39,4 & \\
\hline & M2 & 50,1 & 56,7 & 29,8 & 45,6 & 33,9 \\
\hline \multirow{2}{*}{$\mathrm{Mg}$} & M1 & 17,1 & 11,5 & 13,5 & 11,5 & \\
\hline & M2 & 14,0 & 9,9 & 13,0 & 9,9 & 7,6 \\
\hline
\end{tabular}


de los glucósidos totales en hojas de Stevia hasta el momento de la primera floración, aunque el contenido de rebaudiósido A sí se afectó negativamente ante deficiencias de P, S, K y Cu. Por su parte, la demanda de los principales elementos nutricionales que mostraron Morita 1 y Morita 2 indican que existen requerimientos diferenciales de estos, siendo más exigente el segundo, debido a su mayor contenido de biomasa por planta.

Agradecimientos: Los autores expresan sus agradecimientos a los doctores John Alarcón y Andrés Llanos del Laboratorio de Control de Calidad, de la empresa Prodalysa Ltda., en Chile y Colombia, respectivamente, por su colaboración decidida en el análisis de las muestras de contenido de glucósidos totales. Conflictos de intereses: El manuscrito fue preparado y revisado con la participación de todos los autores, quienes declaramos que no existe ningún conflicto de intereses que ponga en riesgo la validez de los resultados presentados. Financiación: Este estudio fue financiado por la Universidad de Córdoba.

\section{BIBLIOGRAFÍA}

1. ALLAM, A.I.; NASSAR, A.M.; BESHEIT, S.Y. 2001. Nitrogen fertilizer requirements of Stevia rebaudiana (Bert.) Bertoni under Egyptian conditions. Egypt. J. Agric. Res. 79(3):1005-1018.

2. ÁLVAREZ-GARCÍA, R.; TORRES-VALENCIA, J.; ROMÁN, L.; HERNÁNDEZ, J.; CERDA-GARCÍA-ROJAS, C.; JOSEPH-NATHAN, P. 2005. Absolute configuration of the a-methylbutyryl residue in longipinene derivatives from Stevia pilosa. Phytochem. 66:639642 .

3. AZCÓN-BIETO, J.; TALÓN, M. 2008. Fundamentos de Fisiologia Vegetal, $2^{a}$ Ed. McGraw-Hill Interamericana de España. S.A.U. y. Publicacions y Edicions de la Universitat de. Barcelona, 651p.

4. BARATHI, N. 2002. Stevia-A way to sweeten life. Disponible desde internet en: http://www. growmorebiotech.com/article1.htm. (con acceso 11/02/2011).

5. BONDAREV, N.; RESHETNYAK, O.; NOSOV, A. 2003. Effects of nutrient medium composition on development of Stevia rebaudiana shoots cultivated in the roller bioreactor and their production of steviol glycosides. Plant Sci. 165:845-850.

6. BRANDLE, J.E.; TELMER, P.G. 2007. Steviol glycoside biosynthesis. Phytochem. 68:1855-1863.
7. BRANDLE, J.E. 1999. Genetic control of rebaudioside $A$ and $C$ concentration in leaves of the sweet herb, Stevia rebaudiana. Can. J. Plant Sci. 79(1):85-92.

8. BRANDLE, J.E.; RICHMAN, A.; SWANSON, A.; CHAPMAN, B. 2002. Leaf ESTs from Stevia rebaudiana: a resource for gene discovery in diterpene synthesis. Plant Mol. Biol. 50:613-622.

9. CARAKOSTAS, M.C.; CURRY, L.L.; BOILEAU, A.C.; BRUSICK, D.J. 2008. Overview: The history, technical function and safety of rebaudioside A, a naturally occurring steviol glycoside, for use in food and beverages. Food Chem. Tox. 46: S1-S10.

10. DACOME, A.; DA SILVA, C.; DA COSTA, C.; FONTANA, J.; ADELMANN, J.; DA COSTA, S. 2005. Sweet diterpenic glycosides balance of a new cultivar of Stevia rebaudiana (Bert.) Bertoni: Isolation and quantitative distribution by chromatographic, spectroscopic, and electrophoretic methods. Process Biochem. 40:3587-3594.

11. DAS, K.; DANG, R.; SHIVANANDA, T.N.; SEKEROGLU, N. 2007. Influence of bio-fertilizers on the biomass yield and nutrient content in stevia (Stevia rebaudiana. Bert) grown in Indian subtropics. J. Med. Plants Res. 1(1):5-8.

12. DAS, K.; DANG, R.; SHIVANANDA, T.N. 2008. Influence of bio-fertilizers on the availability of nutrients $(\mathrm{N}, \mathrm{P}$ and K) in soil in relation to growth and yield of Stevia rebaudiana grown in South India. Int. J. Appl. Res. Nat.l Prod. 1(1):20-24.

13. DE LIMA, O.; MALAVOLTA, E.; DE SENA, J.; CARNEIRO, J. 1997a. Absorção e Acumulação de Nutrientes em Estévia Stevia rebaudiana (Bert.) Bertoni: I. Macronutrientes. Sci. Agric., Piracicaba. 54(1/2):2330.

14. DE LIMA, O.; MALAVOLTA, E.; YABICO, H. $1997 b$. Influència de estresses nutricionais no teor $\mathrm{e}$ produçâo de esteviosídeo durante o desenvolvimento da Stevia. Pesquisa Agropec. Bras. 32(5):489-494.

15. DE LIMA, O.; MALAVOLTA, E. 1997. Sintomas de desordens nutricionais em estévia Stevia rebaudiana (Bert.) Bertoni. Sci. Agric. 54(1/2):53-61.

16. EPSTEIN, E.; BLOOM, A. 2005. Mineral nutrition of plants: principles and perspectives. Sinauer Associates (EUA). 400p.

17. ERKUCUK, A.; AKGUN, I.H.; YESIL-CELIKTAS, O. 
2009. Supercritical $\mathrm{CO}_{2}$ extraction of glycosides from Stevia rebaudiana leaves: Identification and optimization. J. Supercr. Fluids. 51:29-35.

18. FAN, X.; SHEN, Q.; ZHU, H.; MA, Z.; YIN, X.; MILLER, A. 2005. A comparison of nitrate transport in four different rice (Oryza sativa L.) cultivars. Sci. China (Series C). 48:897-911.

19. FRONZA, D.; FOLEGATTI, M. 2003. Water consumption of the estevia (Stevia rebaudiana (Bert.) Bertoni) crop estimated through microlysimeter. Sci. Agric. 60(3):595-599.

20. GARDANA, C.; SCAGLIANTI, M.; SIMONETTI, P. 2010. Evaluation of steviol and its glycosides in Stevia rebaudiana leaves and commercial sweetener by ultra high performance liquid chromatographymass spectrometry, J. Chromatogr. A, doi:10.1016/j. chroma.2009.12.036.

21. GEUNS, J.M. 2003. Stevioside. Phytochem. 64:913-921.

22. HEARN, L.K.; SUBEDI, P.P. 2006. Determining levels of steviol glycosides in the leaves of Stevia rebaudiana by near infrared reflectance spectroscopy. J. Food Compos. Anal. 22:165-168.

23. JARMA, A. 2008. Estudios de adaptación y manejo integrado de estevia (Stevia rebaudiana Bert.): nueva alternativa agroindustrial del Caribe colombiano. Una revisión. Rev. Col. Sc. Hort. 2(1):109-120.

24. LIU, J.; JIN-WEI, L.; JIAN, T. 2010. Ultrasonically assisted extraction of total carbohydrates from Stevia rebaudiana Bertoni and identification of extracts. Food Bioprod. Process. doi:10.1016/j. fbp.2009.12.005.

25. MARSCHNER, H. 2002. Mineral nutrition of higher plants. $2^{\text {nd }}$ ed. Academic Press. 889p.

26. MIDMORE, D.; RANK, A. 2002. A new rural industry Stevia - to replace imported chemical sweeteners. Rural Industries Research and Development Corporation (RIRDC), Kingston, Australia. 55p.
27. MIZUKAMI, H.; SHIBA, K.; OHASHI, H. 1983. Effect of temperature on growth and stevioside formation of Stevia rebaudiana. Shoyakugaku Zasshi. 37:175179.

28. NEPOVIM, A.; DRAHOSOVA, H.; VALICEK, V.; VANEK, T. 1998. The effect of cultivation conditions on the content of stevioside in Stevia rebaudiana Bertoni plants cultivated in the Czech Republic. Pharmaceut. Pharmacol Lett. 8:19-21.

29. RAMESH, K.; SINGH, V.; MEGEJI, N.W. 2006. Cultivation of stevia [Stevia rebaudiana (Bert.) Bertoni]: A comprehensive review. Adv. in Agron. 89:137-177.

30. RICHMAN, A.; SWANSON, A.; HUMPHREY, T.; CHAPMAN, R.; MCGARVEY, B.; POCS, R.; BRANDLE, J. 2005. Functional genomics uncovers three glucosyltransferases involved in the synthesis of the major sweet glucosides of Stevia rebaudiana. Plant J. 41:56-67.

31. SRIVASTAVA, L.M. 2002. Plant growth and development. Hormones and environment. Academic Press, Amsterdam. 772p.

32. SU, J.; XIAO, M.; LI, Q.; LIU, B.; TONG, T. 2006. Mapping QTLs for phosphorus-deficiency tolerance at wheat seedling stage. Plant and Soil. 281(1-2):25-36.

33. TAIZ, L.; ZEIGER, E. 2006. Plant Physiology. IV ed. Sinauer Associates, Inc. p.75-83.

34. UTUMI, M.; MONNERAT, P.; GOMES, P.; RESENDE, P.; CAMPOS, V. 1999. Deficiência de macronutrientes em estévia: Sintomas visuais e efeitos no crescimento, Composição química e produção de esteviosídeo. Pesq. Agropec. Bras. 34(6):1039-1043.

35. WHITE, P.; BROADLEY, M. 2003. Calcium in plants. Ann. Bot. 92:487-511.

Recibido: Mayo 10 de 2011

Aceptado: Marzo 4 de 2012 